\title{
Adipose-derived mesenchymal stem cell-loaded $\beta$-chitin nanofiber hydrogel promote wound healing in rats
}

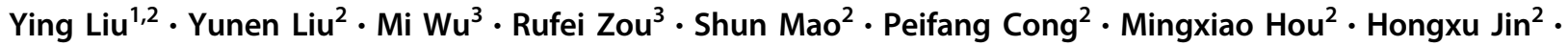 \\ Yan $\mathrm{Zhao}^{3} \cdot$ Yongli Bao ${ }^{1}$
}

Received: 25 July 2021 / Accepted: 28 November 2021 / Published online: 20 January 2022

(c) The Author(s) 2022

\begin{abstract}
Because of stem cells are limited by the low efficiency of their cell homing and survival in vivo, cell delivery systems and scaffolds have attracted a great deal of attention for stem cells' successful clinical practice. $\beta$-chitin nanofibers $(\beta$-ChNF) were prepared from squid pens in this study. Fourier transform infrared spectroscopy, X-ray diffraction and scanning electron microscopy proved that $\beta$-ChNFs with the diameter of 5 to $10 \mathrm{~nm}$ were prepared. $\beta$-ChNF dispersion became gelled upon the addition of cell culture medium. Cell culture experiments showed that $\beta$-ChNFs exhibited negligible cytotoxicity towards ADSCs and L929 cells, and it was found that more exosomes were secreted by the globular ADSCs grown in the $\beta$-ChNF hydrogel. The vivo experiments of rats showed that the ADSCs-loaded $\beta$-ChNF hydrogel could directly cover the wound surface and significantly accelerate the wound healing and promote the generation of epithelization, granulation tissue and collagen. In addition, the ADSCs-loaded $\beta$-ChNF hydrogel clearly regulated the expressions of VEGFR, $\alpha$-SMA, collagen I and collagen III. Finally, we showed that ADSCs-loaded $\beta$-ChNF hydrogel activated the TGF $\beta /$ smad signaling. The neutralization of TGF $\beta$ markedly reduced Smad phosphorylation and the expressions of TIMP1, VEGFR and $\alpha$-SMA. Taken together, these findings suggest that ADSCs-loaded $\beta$-ChNF hydrogel promises for treating wounds that are challenge to heal via conventional methods.
\end{abstract}

\section{Graphical abstract}

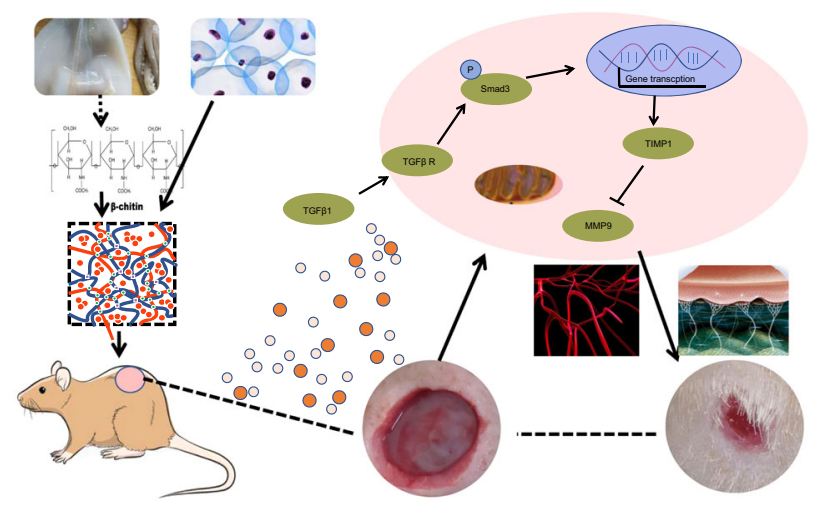

Hongxu Jin

cszx_jhx@163.com

Yan Zhao

zhaoyan@jihualab.com

$\triangle$ Yongli Bao

baoy1800@nenu.edu.cn
1 National Engineering Laboratory for Druggable Gene and Protein Screening, Northeast Normal University, Changchun 130117, China

2 Emergency Medicine Department of General Hospital of Northern Theater Command, Laboratory of Rescue Center of Severe Wound and Trauma PLA, Shenyang 110016, China

3 Jihua Laboratory, Foshan 528200, China 


\section{Introduction}

As a soft tissue that covers the entire surface area of the body, skin is mainly responsible for protecting various tissues and organs from physical, mechanical, chemical and pathogenic microorganisms [1]. Wound, as one of the most commonly encountered healthcare issue, could induce significant distress to patients by providing a portal of entry into the body for pathogens, and their appropriate management is critical for the wellbeing of affected individuals $[2,3]$. Wound healing includes the granulation tissue formation, scar tissue formation and other synergistic effects. During the healing process, numerous distinct cells, especially stem cell are activated, recruited into the wound, and secreted various growth factors and cytokines [4-6]. With the development of regenerative medicine, stem cells are becoming new strategies for effective wound treatment $[7,8]$.

The abundant supply of fat tissue as well as the ease of isolation, proliferation and secretion of growth factors related to wound healing, makes adipose-derived mesenchymal stem cells (ADSCs) an ideal cell type for non-healing wound treatment. ADSCs can be differentiated into different lineages and secrete paracrine factors to initiate the tissue regeneration process [9]. Currently, intravenous infusion and local injection are the major routes for ADSC administration, which are limited by the low efficiency of their cell homing and survival in vivo during clinical application [10]. Therefore, the efficacy of cell therapy significantly depends on whether cellular carriers could successfully deliver therapeutic cells to target tissues and sites, as well as the surrounding microenvironment $[11,12]$.

By incorporating therapeutic cells, cell delivery systems provide a cell-friendly environment that can be placed at the target site, and it showed that ADSCs combined with different scaffolds could noticeably improve wound healing [13]. To date, cell delivery systems and scaffolds have attracted a large amount of attention, especially cellular scaffolds composed of various synthetic and natural polymers. Typically be prepared under mild processing conditions, hydrogel is a kind of polymer network system with a certain shape. They have soft viscoelastic properties and are similar in function and structure to extracellular matrix (ECM). Hydrogels have been widely used in the tissue engineering and regenerative medicine fields because of their various properties as well as supporting the incorporation of therapeutic cells $[14,15]$. Chitin is the second most abundant polysaccharide in nature after cellulose and is widely found in the shells of shrimps, crabs, insects and other crustaceans, as well as the cell walls of fungi and bacteria. It has already been used in suture line, artificial dialysis membrane, tissue engineering and wound repair
[16]. Nanofibrous structures of chitin nanofibers are excellent candidates as wound dressing materials: their porous structure simulates the fibrous structure of natural extracellular matrix; facilitates cell seeding; allows diffusion of nutrients, oxygen and waste; and can absorb wound exudates, to prevent excessive dehydration and bacterial infection from the wound [17]. Chitin exists as typically two different forms in nature, namely $\alpha$ and $\beta$. Formed by two symmetrically and alternately antiparallel chitin chains, $\alpha$ chitin has higher degree of intermolecular hydrogen bonding and a more compact and stable structure. While $\beta$-chitin has intrasheet hydrogen-bonding by parallel chains with weaker intermolecular interactions between the chains and higher chemical reactivity. Kangquan Shou et al has found that $\alpha$-chitin nanofibers hydrogel could affect bone marrow mesenchymal stem cell (BMSC) differentiation and exhibit better viability of the cells than local injection of BMSC in wound treatments [18]. However, the characteristics of $\beta$-chitin nanofibers in ADSCs cell culture, and their effect on exosome secretion and wound healing remain to be further studied.

In this work, $\beta$-chitin nanofibers ( $\beta$-ChNF) were obtained from squid cartilage, and used to prepare scaffold carriers for stem cells application. Following toxicity evaluation and the determination of successful stem cells growth in the scaffold, rat wound models were established to evaluate the wound healing effect and mechanism of the dressing. The results provide new perspectives for the application of stem cells by using $\beta$-ChNF hydrogel as the scaffold for full-thickness cutaneous wound healing.

\section{Materials and methods}

\subsection{Materials}

Squid pens were obtained from a local fish market, China. Hydrochloric acid, sodium hydroxide and acetic acid were purchased from Sinopharm Chemical Reagent Co., Ltd., China. ADSCs (MUBMD-01001) and adipose-derived mesenchymal stem cell medium (MUBMD-03011-440) were purchased from Cyagen Biosciences, China. L929 (BFN60805937) cells were provided by Cobioer, China. Masson's staining kit was purchased from Sigma, USA.

\subsection{Preparation of $\beta$-chitin}

$\beta$-chitin was prepared from squid pens through demineralization and deproteinization steps. Briefly, $50 \mathrm{~g}$ of powdered squid pen was washed and dried, and dispersed in $750 \mathrm{~mL}$ of $0.1 \mathrm{~mol} / \mathrm{L}$ hydrochloric acid solution for $20 \mathrm{~h}$ for demineralization. After washing with D.I. water to neutrality, squid pens were further treated with $750 \mathrm{~mL}$ of $4 \mathrm{wt}$ 
$\%$ sodium hydroxide solution for $10 \mathrm{~h}$ at $80{ }^{\circ} \mathrm{C}$ for deproteinization and washed with D.I. water at a resistivity of $18.2 \mathrm{M} \Omega \bullet \mathrm{cm}$ to neutrality.

\subsection{Gelation of $\beta$-chitin nanofiber ( $\beta$-ChNF) dispersion}

A $\beta$-ChNF dispersion was prepared by adding the purified $\beta$-chitin to D.I. water at a concentration of $0.3 \mathrm{wt} \%$, acidified to $\mathrm{pH} 3$ with acetic acid, and treated by the ultrasonic homogenizer (Scientz JY92-IIDN, China) in an ice-water bath for $6 \mathrm{~min}$ at $19.5 \mathrm{kHz}$ and $300 \mathrm{~W}$. Gelation of the $\beta$-ChNF dispersion occurred when culture medium was added to the dispersion at a ratio of 3:1 $\beta$-ChNF dispersion: medium. The formation of $\beta$-chitin was confirmed by Fourier transform infrared (FTIR) spectroscopy (Agilent Technologies Cary 630 spectrometer). The sample's crystallinity was characterized by X-ray diffraction (XRD; Max2500PC, Rigaku D, Japan). The morphology of the $\beta$-ChNF dispersion before and after gelation was observed through scanning electron microscopy (SEM, inspect F50, FEI). The rheological characterization of the $\beta$-ChNF dispersion with and without added culture medium was conducted via a rheometer (HAAKE MARS40, Germany).

\subsection{Cell culture and cytotoxicity}

ADSCs were cultured in adipose-derived mesenchymal stem cell medium that contained $10 \%$ fetal bovine serum, $1 \%$ penicillin-streptomycin (T180125G001), and 1\% glutamine (T180516G001). For the hydrogel cell culture, cell medium was added to the $\beta$-ChNF solution at a 3:1 volume ratio, respectively, and allowed to gel for $15 \mathrm{~min}$ at room temperature, then $1 \times 10^{6}$ ADSCs were added. L929 cells were cultured in MEM cell medium (CPB50009) containing $10 \%$ horse serum, 1\% penicillin-streptomycin (CPB50027) and 1\% glutamine (CPB50028). According to ISO 10993 part 5 guidelines (ISO document 10993, 1992), the cytotoxicity of the $\beta$-ChNF hydrogel was evaluated by an extraction test and 3-(4,5-dimethylthiazol-2-yl)-2,5-diphenyltetrazolium bromide (MTT) assay of ADSCs and L929 cells. The cells were seeded in 96-well plates and cultured in $37^{\circ} \mathrm{C}$ for $24 \mathrm{~h}$ with $5 \% \mathrm{CO}_{2}$. The medium in hydrogel group was replaced with the extracts. The control group was treated with normal culture media. After culturing for a further 12,24 , or $48 \mathrm{~h}, 20 \mu \mathrm{L}$ of MTT $(5 \mathrm{mg} / \mathrm{mL}$ in PBS) was added to each well. $4 \mathrm{~h}$ later, the medium containing MTT was replaced with $150 \mu \mathrm{L}$ of dimethyl sulfoxide (DMSO). After incubation for $15 \mathrm{~min}$ with agitation, the absorbance of the DMSO solution at $490 \mathrm{~nm}$ was measured using a plate reader (BIO-RAD iMark). Cell growth in the scaffold was observed via an inverted phase contrast microscope (TS100, Nikon, Japan). Exosome secretion was detected by transmission electron microscopy (G2 spititi FEI, Tecnai) and nanoparticle tracking analysis (ZetaView_Particle Metrix, ZetaView PMX 110, Particle Metrix). During the nanoparticle tracking analysis, the sample pool was cleaned with deionized water and the instrument was calibrated with polystyrene microsphere $(110 \mathrm{~nm})$. After that, 1X PBS buffer (Biological Industries, Israel) was used to clean the sample pool and the sample was diluted with $1 \mathrm{X}$ PBS buffer (BI, Israel) to be checked.

\subsection{Animals}

Sprague-Dawley rats (6-8 weeks) were purchased from Liaoning Changsheng Biotechnology Co., Ltd. and were kept in their cages maintaining at $22 \pm 3{ }^{\circ} \mathrm{C}$ with a humidity of $45-60 \%$. They were all allowed free access to food and water. After acclimation, all rats were randomly divided into the control, hydrogel, ADSCs and ADSCs+hydrogel groups. Animal experiments were approved by the Ethics Committee of the General Hospital of the Northern Theater Command.

\subsection{Wound healing activity}

The full-thickness defect wound model was established on the basis of a procedure used in a previous report [19]. Circular full-thickness cutaneous wounds were created and carefully observed $0,2,4,7,8$ and 10 days post-surgery. The wound was photographed and the area was measured by Image $\mathbf{J}$ (National Institutes of Health, USA). The wound healing rate was calculated as follows: wound healing rate $(\%)=(\mathrm{A} 0-\mathrm{At}) / \mathrm{A} 0 \times 100 \%$.

\subsection{Histological examination}

Ten days after surgery, part of the rat skin samples were harvested and fixed in 10\% formaldehyde. For histological staining, $5-\mu \mathrm{m}$-thick sections were sliced and stained using hematoxylin and eosin (H\&E) staining. To assess the amount of generated collagen, sections were stained with masson's staining kit (Sigma, USA). Image J software was used to detect the percentage of the collagen staining area (blue) in the total area. Immunofluorescence staining was carried out to localize VEGFR and $\alpha$-SMA. Briefly, skin tissues were dewaxed and the endogenous peroxidase was inactivated. After antigen retrieval, the samples were blocked with BSA, incubated with primary antibody and a fluorescent secondary antibody.

\subsection{Western blot}

The protein for western blot analysis was extracted from skin tissues and concentration was determined using a BCA 
protein assay kit (Cat No: FD2001, Hangzhou Fude Biological Technology Company, China). The primary antibody included VEGFR (1:1000, ab39638, Abcam, UK), $\alpha$ SMA (1:1000, ab124964, Abcam, UK), collagen I (1:1000, ab270993, Abcam, UK), collagen III (1:1000, ab7778, Abcam, UK), TGF $\beta 1$ (1:2000, ab215715, Abcam, UK), Smad3 (1:2000, ab40854, Abcam, UK), Smad2 (1:2000, ab40855, Abcam, UK), P-smad3 (phospho T179, 1:2000, ab74062, Abcam, UK), P-smad2 (phospho S467, 1:2000, ab280888, Abcam, UK), TIMP1 (1:2000, ab216432, Abcam, UK), GAPDH (1:4000, 2118, Cell Signaling Technology, Boston, USA) and Anti-rabbit secondary antibody (1:4000, ab6721, Abcam, UK). Proteins were visualized through a Clarity TM Western ECL Substrate (170-5061; Bio-Rad Laboratories Inc., USA) and a Tanon 5200 fully automatic chemiluminescence image analysis system (Tanon Science and Technology Co. Ltd., Shanghai, China).

\subsection{Blockade of TGF $\beta 1$}

One day after treatment with ADSCs-loaded $\beta$-ChNF hydrogel, we hypodermic injected a neutralizing antibody (MAB 1835, R\&D Systems, Minneapolis, MD) in a single dose of $400 \mu \mathrm{g} / \mathrm{kg}$ to a group of rats. Five days after the injection, the protein expression of TGF $\beta 1$, P-smad3, P-smad2, Smad3, Smad2, TIMP1, VEGFR and $\alpha$-SMA in rats were further detected by western blot.

\subsection{Statistical analysis}

All data were analyzed by SPSS 22.0 (IBM, NY, USA). Quantitative data were expressed as mean \pm standard deviation. The two-tailed paired Student's $t$ test was used for group comparisons. $P$ values $<0.05$ were considered statistically significant. The correlation analysis between the concentration of VEGFR and the number of blood vessels was conducted by pearson test.

\section{Results}

\subsection{Characterization of the porous chitin nanofiber hydrogel}

Figure 1A shows the preparation process of $\beta$-chitin nanofiber with the degree of deacetylation of about $10 \%$ and a uniform width of 5-10 $\mathrm{nm}$. To identity the physicochemical structure of the as-prepared chitin nanofiber, FTIR and XRD measurements have been carried out. As shown in Fig. 1B, the characteristic peaks of chitin nanofibers occurred at $3434 \mathrm{~cm}^{-1}, 3270 \mathrm{~cm}^{-1}$, which could be assigned to the stretching vibration of $\mathrm{O}-\mathrm{H}$ and $\mathrm{N}-\mathrm{H}$, respectively [20, 21]. In particular, the adsorption bands involving $1628 \mathrm{~cm}^{-1}$ (amide I) and $1550 \mathrm{~cm}^{-1}$ (amide II) suggested that a $\beta$-chitin structure was prepared from squid pen $[22,23]$. As shown in XRD pattern (Fig. 1C), the main diffraction peaks of chitin nanofibers appeared at $8.6^{\circ}(020$ plane) and $19.6^{\circ}$ (110 plane), further indicating the crystal structure of $\beta$-chitin [21]. Figure 1D and E shows the hydrogel before and after the addition of cell culture medium, respectively. The addition of cell culture medium initiated gel formation. Figure 1F, G shows SEM images of the chitin nanofiber and the morphological characteristics in presence of cell culture medium, respectively. The intermediate pore size of the hydrogels was $\sim 200 \mu \mathrm{m}$. Figure $1 \mathrm{H}$ shows the gelling kinetics based on measuring $\mathrm{G}^{\prime}$ and $\mathrm{G}^{\prime \prime}$ as a function of time. Both $G^{\prime}$ and $G$ ' of the hydrogel increased significantly when cell culture medium was added, indicating a stronger hydrogel was formed. Figure 1I shows the results of the MTT assay, the viabilities of ADSCs and L929 cells cultured in the chitin membrane were more than $95 \%$. These in vitro cytotoxicity results indicated that $\beta$-chitin had low cell toxicity and was applicable in cell culture.

\subsection{Preparation of ADSC-loaded $\beta$-ChNF hydrogel}

$\beta$-ChNF hydrogel dispersion $(500 \mu \mathrm{L})$ and C57BL/6 mouse ADSCs medium $(1500 \mu \mathrm{L})$ were added to an ultra-low attachment 24-well plate. After $15 \mathrm{~min}$, the medium was discarded and $1000 \mu \mathrm{L}$ of C57BL/6 mouse ADSCs medium containing $1 \times 10^{6}$ ADSCs was added (Fig. 2A). The microstructure of ADSCs-loaded $\beta$-ChNF hydrogel is shown in Fig. 2. Microscopy revealed that the ADSCs grew as globules (Fig. 2B) and the GFP-labeled ADSCs exhibited green fluorescence under a fluorescence microscope (Fig. 2C). The cell distribution was observed by scanning electron microscopy. The ADSCs-loaded $\beta$-ChNF hydrogel exhibited a highly porous and interconnected interior structure that supported cell growth (Fig. 2D, E). Furthermore, transmission electron microscopy was used to detect exosomes in the cell supernatant (Fig. 2F, G). Nanoparticle tracking analysis showed that there were more ADSC exosomes in the hydrogel cell culture than those in the control cell culture (Fig. 2H, I).

\subsection{ADSC-loaded $\beta$-ChNF hydrogels significantly increased the wound healing rate}

Rats in each group were in good condition. There was no significant difference in the body weight of rats among the groups. Two days after surgery, the wounds in all groups were dry, and no obvious infection, edema or inflammatory exudation was observed in the wounds or surrounding skin tissue. Seven days after surgery, the wound in the control 
A

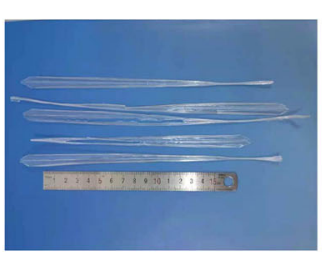

Squid cartilage

$B$

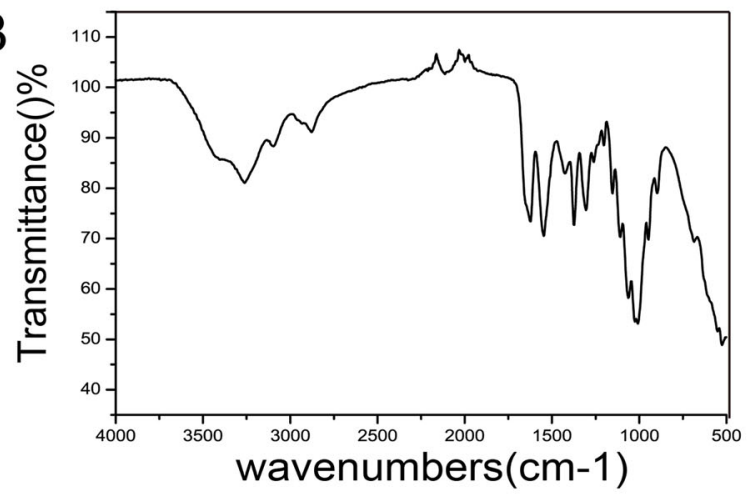

C

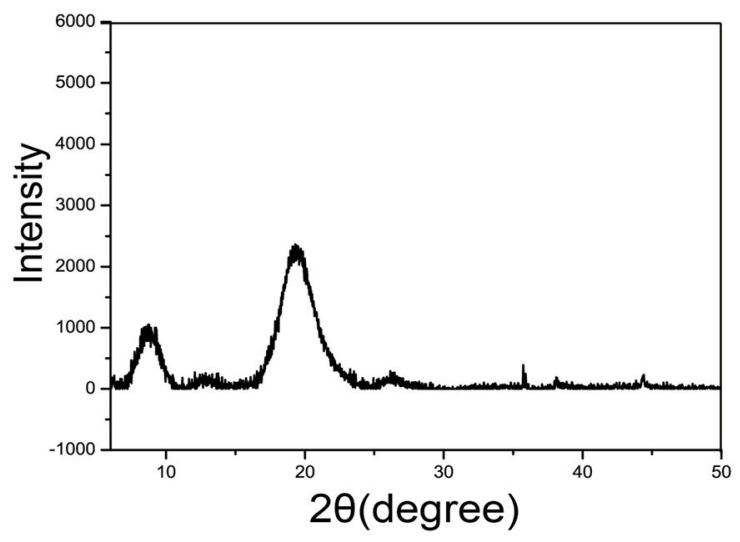

G

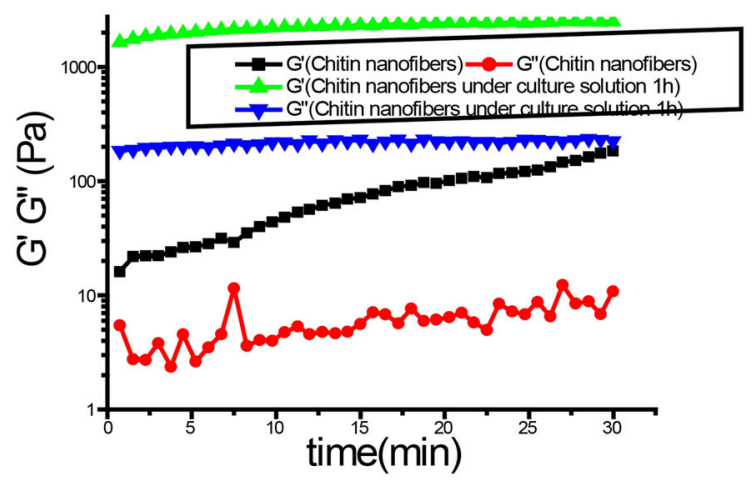

Fig. 1 The characterization of $\beta$-chitin nanofibers hydrogel. A The sketch map of $\beta$-chitin nanofibers preparated from squid cartilage. B FTIR spectra and (C) XRD profile suggested a $\beta$-chitin structure. D Representative digital photographs of the $\beta$-chitin nanofibers hydrogel before and after the reaction with cell culture medium. E SEM image

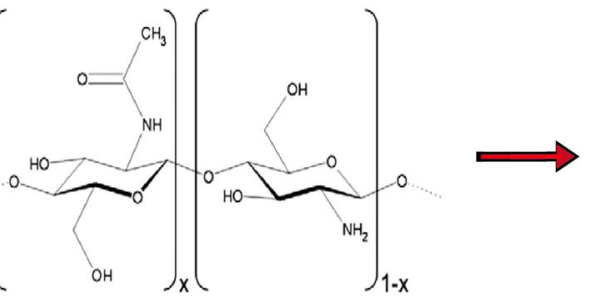

$\beta$-chitin

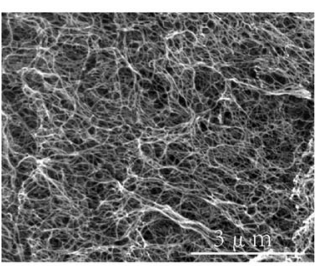

Nanofiber

\section{$\mathrm{D}$ \\ $\mathrm{E}$}
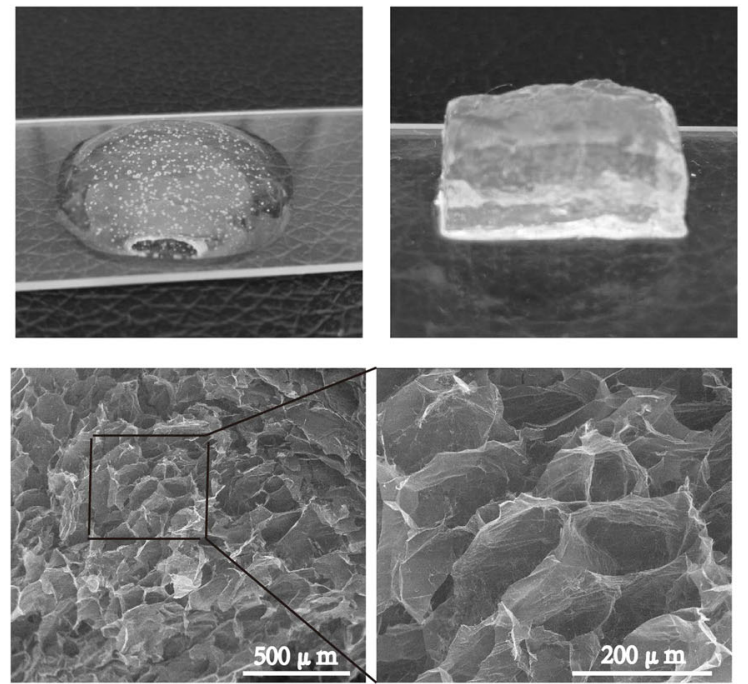

$\mathrm{F}$

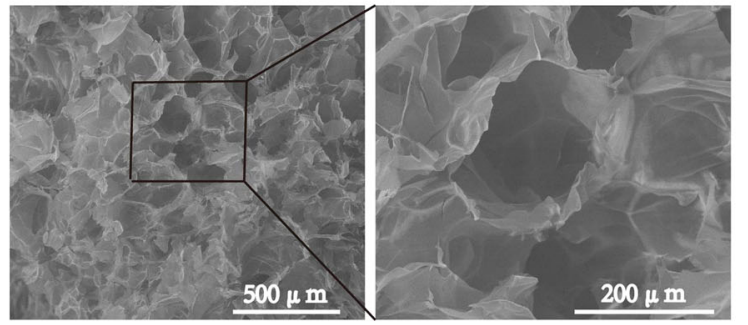

$\mathrm{H}$

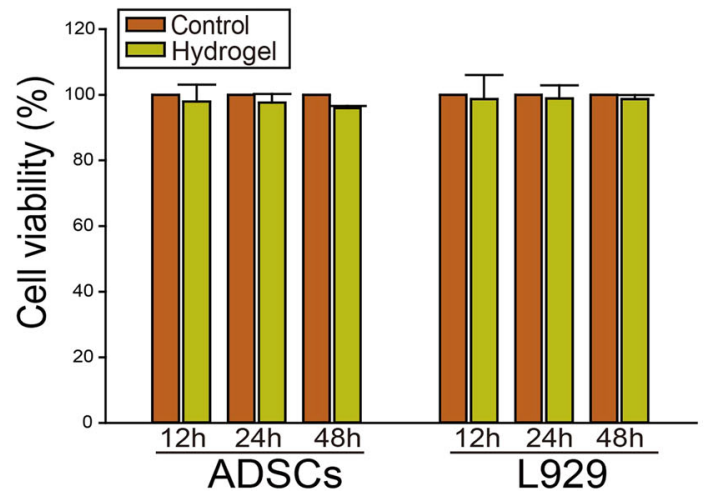

of the chitin hydrogel. F SEM image of the $\beta$-chitin hydrogel in the presence of culture solution. G Storage modulus $\left(\mathrm{G}^{\prime}\right)$ and loss modulus $\left(\mathrm{G}^{\prime \prime}\right)$-strain dependence of chitin nanofibers and chitin nanofibers in the presence of culture solution. $\mathbf{H}$ The extracts of $\beta$-chitin membrane incubated with ADSCs and L929 cells shown no cytotoxicity 
A

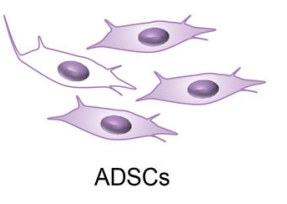

$+$
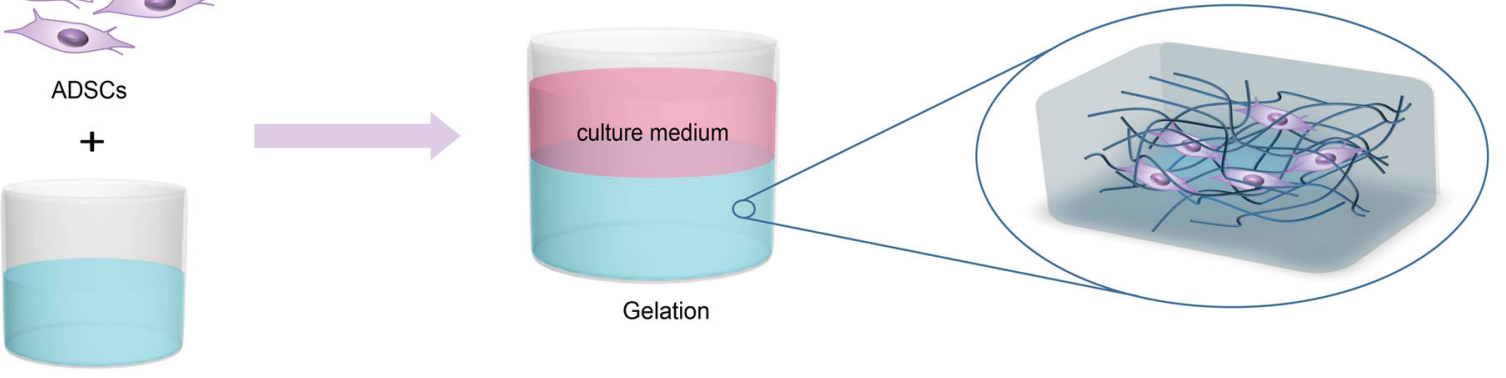

$\beta$-ChNF dispersion
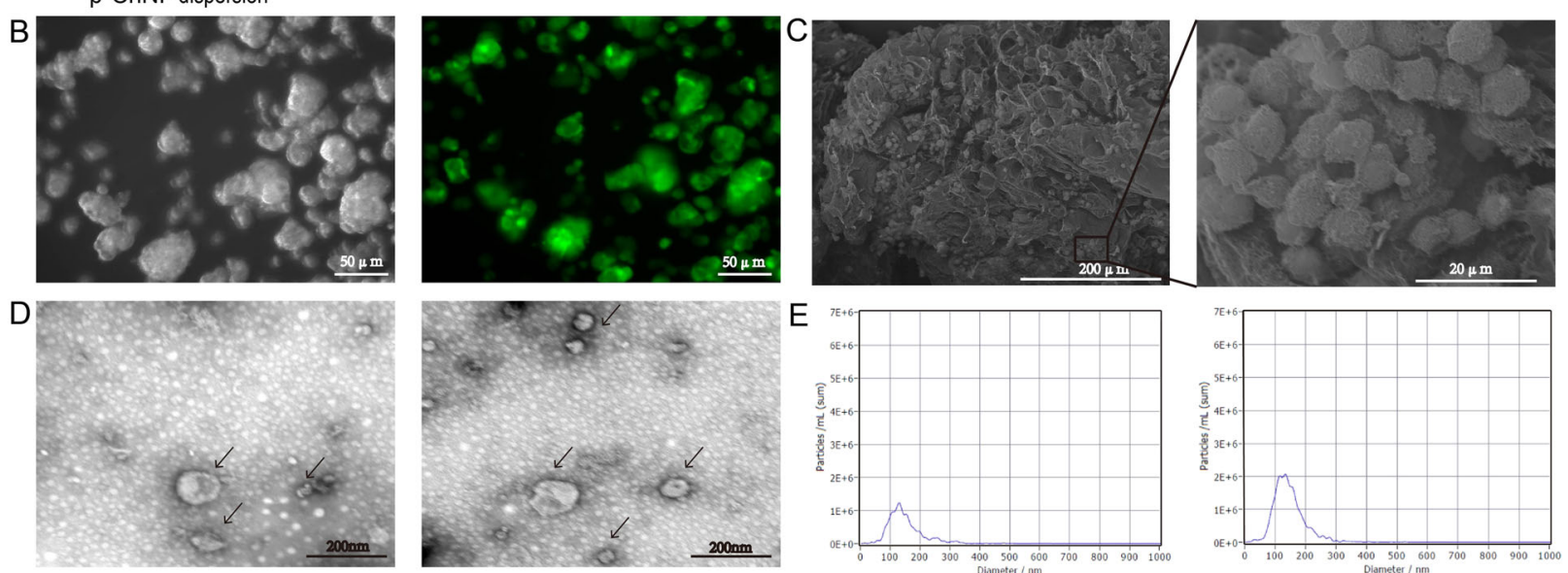

$\mathrm{E}$
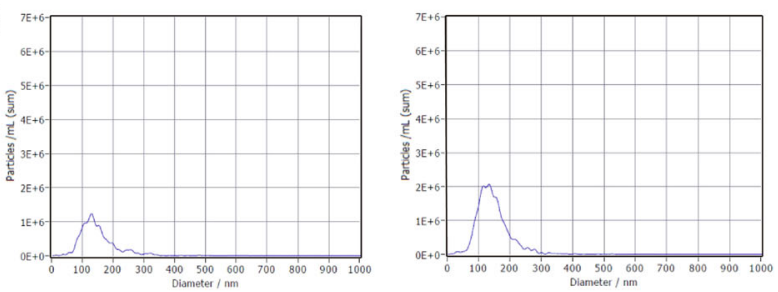

Fig. 2 Growth of ADSCs in $\beta$-chitin hydrogel. A The stepwise diagram for preparation of ADSCs-loaded $\beta$-ChNF hydrogel. B Globular ADSCs grown in the $\beta$-chitin hydrogel scaffold, and the microexamination proved the growth of GFP-labeled ADSCs. C SEM images of the ADSCs-loaded $\beta$-ChNF hydrogel. D Transmission

electron microscopy proved the secretion of exosomes in normal or $\beta$-chitin hydrogel culture (Left, the control group. Right, the hydrogel group). E Nanoparticle tracking analysis of exosomes in normal or $\beta$-chitin hydrogel culture (Left, the control group. Right, the hydrogel group)

group was hard, while the texture of the wounds in the hydrogel and ADSCs+hydrogel groups remained soft. In addition, the wounds in the ADSCs+hydrogel group were markedly smaller than those in the control, ADSCs or hydrogel groups. Image $\mathbf{J}$ software was used to measure the wound healing rate of the full-thickness wounds in each group. The results showed: compared with the control, ADSCs and hydrogel groups; the ADSCs-loaded $\beta$-ChNF hydrogel significantly increased the wound healing rate (Fig. 3, $P<0.05$ ). Seven days after surgery, the wound healing rate of the ADSCs+hydrogel group was nearly $70 \%$.

\subsection{ADSC-loaded $\beta$-ChNF hydrogels improved the pathological changes of wound in rats}

Histological examination showed poor granulation, incomplete healing, no epithelium formation and fewer capillaries in the control group; bleeding, necrosis, and scab were still present in the ADSCs and hydrogel groups; compared with the control group, the healing was

significantly increased, the epithelialization rate was higher, and the granulation tissue and capillary formation were increased in the hydrogel, ADSCs or ADSCs +hydrogel group; compared with the hydrogel or ADSCs group, the epithelialization rate in the ADSCs+hydrogel group was also increased (Fig. 4A, $P<0.05$ ); these were consistent with the above wound healing results. Furthermore, the hydrogel group rats showed better epithelialization than ADSCs group mice (Fig. 4A, $P<0.05$ ). Modified Masson staining showed that the amount of collagen deposited $10 \mathrm{~d}$ after surgery in the hydrogel, ADSCs or ADSCs+hydrogel group was markedly higher than that in the control group; when compared with the ADSCs group, the collagen area in the ADSCs+hydrogel group was also increased (Fig. 4B, $P<0.05$ ). These data suggested that ADSCs-loaded $\beta$-ChNF hydrogel showed the combined effects of hydrogels and ADSCs to accelerate wound healing in rats. ADSCs-loaded $\beta$-ChNF hydrogel took the advantage of hydrogel by accelerating epithelialization, and at the same time took the advantage of stem cell therapy by increasing collagen production. 
Fig. 3 ADSCs-loaded $\beta$-ChNF hydrogel increased the wound healing rate in rats. A

Photographs of wounds closure from 0 to $10 \mathrm{~d}$ after surgery. B Weight shown no changes during the treatment period. C The wound healing rate of full-thickness wounds for each treatment group. ${ }^{*} P<0.05$, vs. the control group

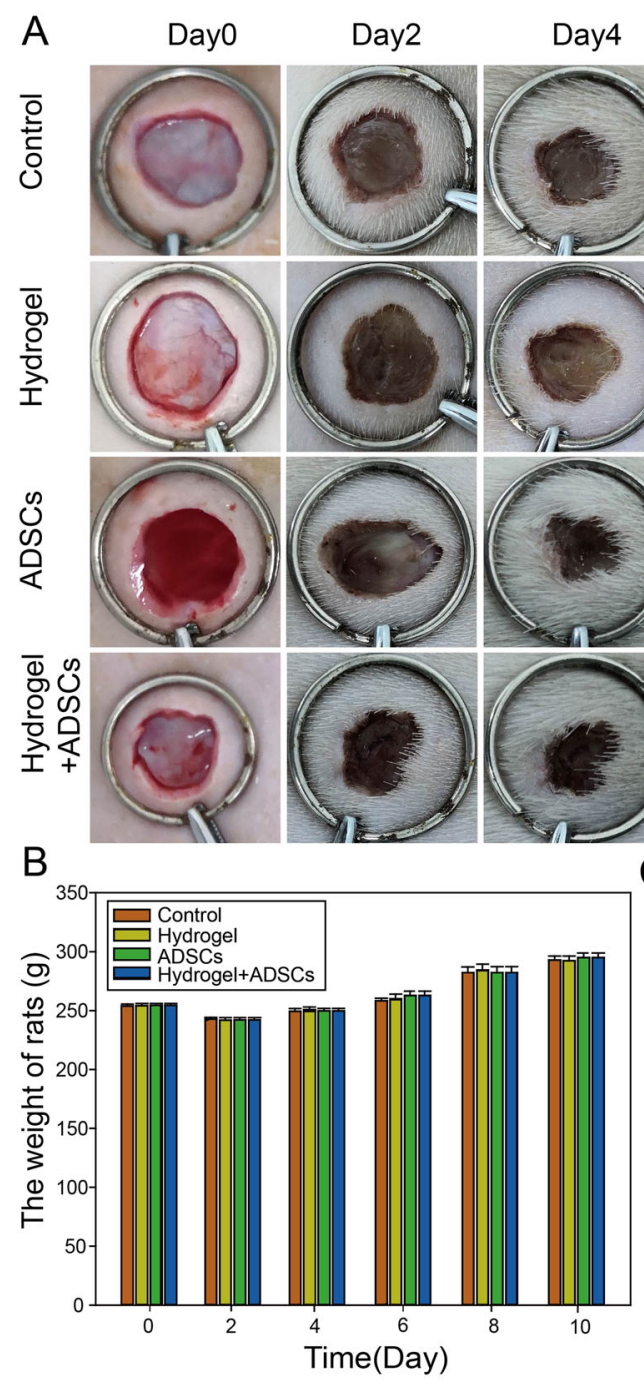

C
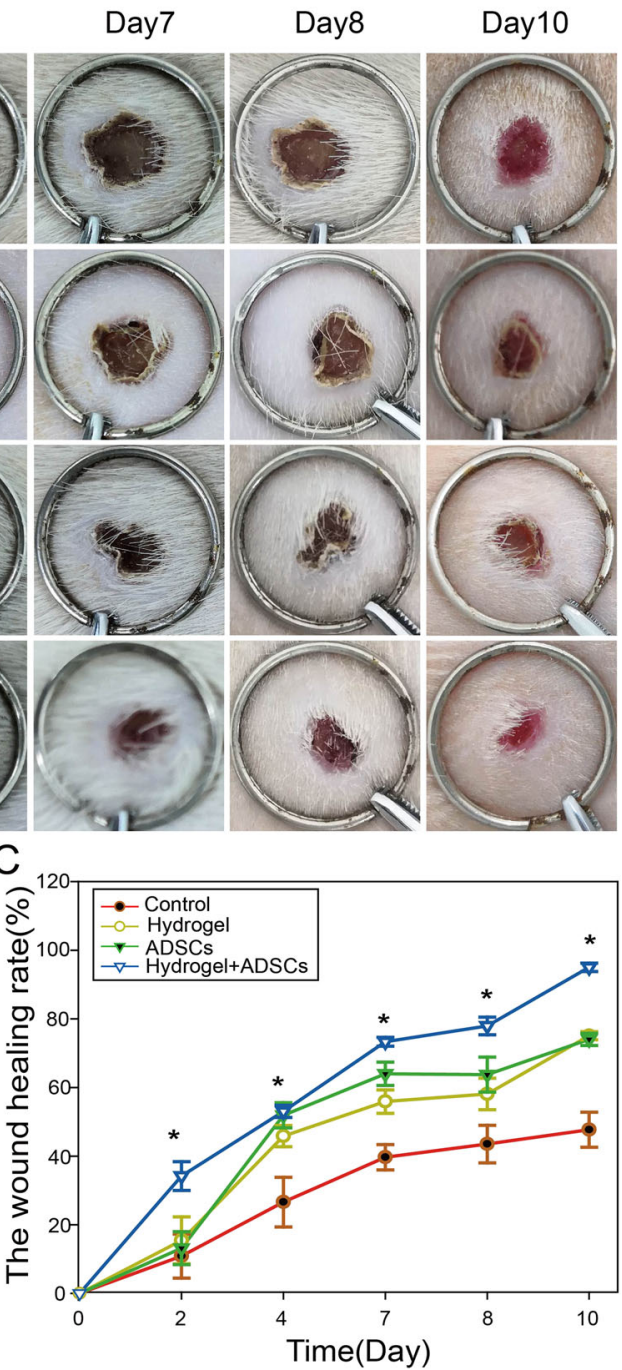

\subsection{ADSC-loaded $\beta$-ChNF hydrogels accelerated collagen deposition and angiogenesis}

Western blot showed that the expressions of VEGFR, $\alpha$ SMA, collagen I and collagen III of the hydrogel, ADSCs or ADSCs+hydrogel group were significantly higher than those of the control group $10 \mathrm{~d}$ after surgery; ADSCsloaded $\beta$-ChNF hydrogel accelerated the expression of VEGFR and $\alpha$-SMA when compared with hydrogel or ADSCs treatment; the ADSCs group mice also showed the higher expression of VEGFR and $\alpha$-SMA than the hydrogel group mice $(P<0.05)$. Furthermore, ADSCs-loaded $\beta$-ChNF hydrogel group mice also showed higher expression of collagen III than ADSCs mice (Fig. 5A, B, $P<0.05)$. This indicated that ADSCs-loaded $\beta$-ChNF hydrogel promoted angiogenesis and collagenation in wound healing. ADSCs-loaded $\beta$-ChNF hydrogel was better for the expression of collagen III than hydrogel, which was good for preventing scar formation in wound healing.
Immunofluorescence staining showed that the expression of VEGFR and $\alpha$-SMA in the ADSCs+hydrogel group was significantly higher than that of the control group $10 \mathrm{~d}$ after surgery (Fig. 5C, $P<0.05$ ). The number of blood vessels was positive correlation with the VEGFR concentration $(r=0.8499)$.

\subsection{ADSC-loaded $\beta$-ChNF hydrogels regulated the TGF $\beta /$ smad signaling pathway}

TGF $\beta$ can be regulated by mesenchymal stem cells and result in the phosphorylation of Smad2 and Smad3. These will trigger target gene transcription to play an active role in the wound healing process, collagenation as well as neoangiogenesis. To further clarify the mechanism of ADSC-loaded $\beta$-ChNF hydrogel on the wound, we detected the expression of TGF $\beta /$ smad signaling pathway. Western blot showed that the expressions of TGF $\beta 1$, P-smad3, P-smad2 and TIMP1 in the ADSCs and ADSCs+hydrogel 

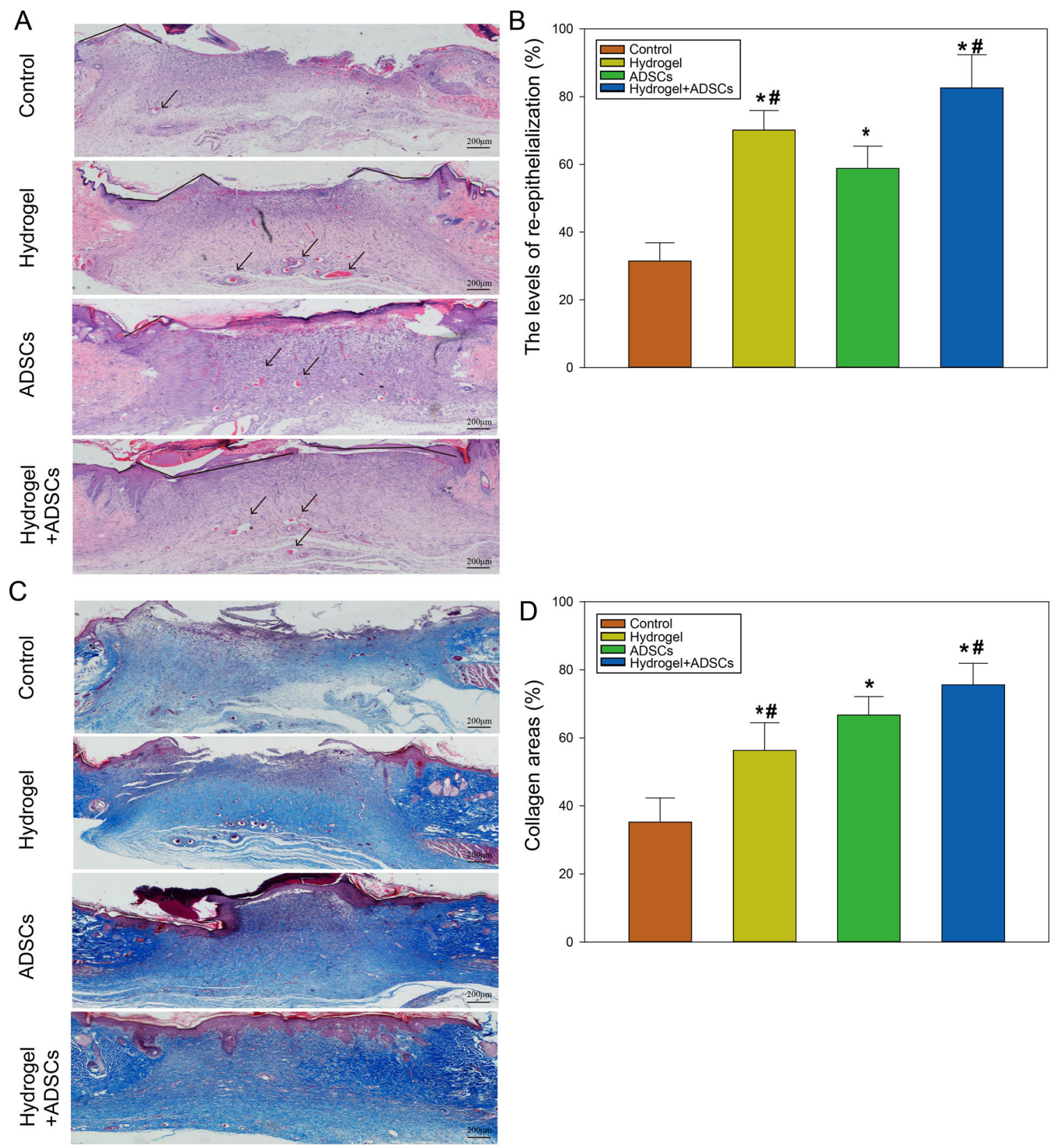

Fig. 4 ADSCs-loaded $\beta$-ChNF hydrogel accelerated wound healing and collagen deposition in rats. A Representative H\&E staining images of each group. The red arrows indicated new blood vessels. B Image J software was used to measure the levels of re-epithelialization of the full-thickness wounds in each group. ${ }^{*} P<0.05$, vs. the control group; ${ }^{\#} P<0.05$, vs. the ADSCs group; ${ }^{\circledR} P<0.05$, vs. the ADSCs + hydrogel

group. C Representative modified Masson staining images of each group. D Image $\mathbf{J}$ software was used to measure the collagen deposition of the full-thickness wounds in each group. ${ }^{*} P<0.05$, vs. the control group; ${ }^{\#} P<0.05$, vs. the ADSCs group; ${ }^{\circledR} P<0.05$, vs. the ADSCs+hydrogel group

groups were significantly increased at $10 \mathrm{~d}$ after surgery than those of the control group $(P<0.05)$, while there was no significant difference in the hydrogel group $(P>0.05)$. The expressions of TGF $\beta 1$, P-smad3, P-smad2 and TIMP1 in the ADSCs+hydrogel group were higher than those of the ADSCs group (Fig. 6A, $P<0.05$ ). To prove the rule of $\mathrm{TGF} \beta / \mathrm{smad}$ in wound healing, we further detected the

expression of VEGFR and $\alpha$-SMA after blockade of TGF $\beta 1$. The results showed that the expression of VEGFR and $\alpha$-SMA in ADSCs+hydrogel group were lower after neutralizing antibody treatment (Fig. $6 \mathrm{~B}, P<0.05$ ). These date suggested that ADSC-loaded $\beta$-ChNF hydrogel and ADSCs could regulate the TGF $\beta /$ smad signaling pathway to promote neoangiogenesis. 

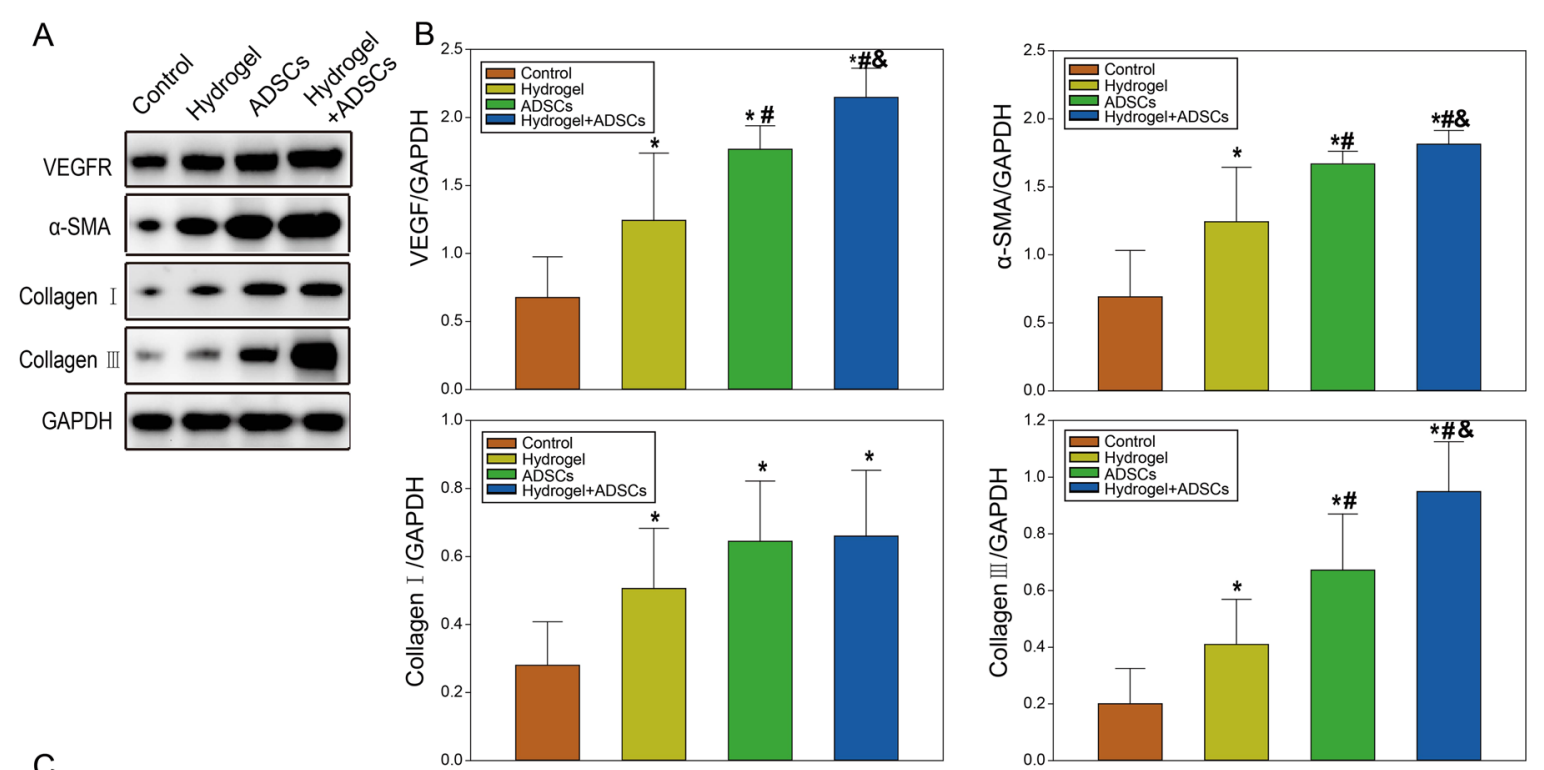

C

VEGFR

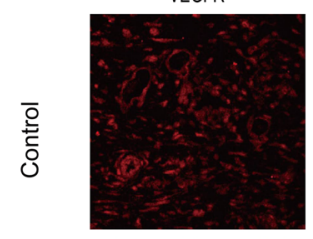

DAPI
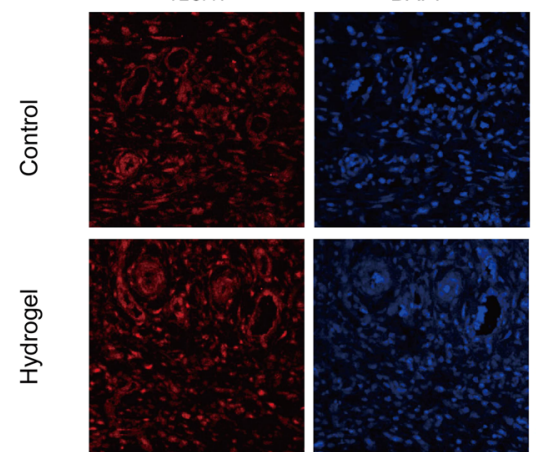

倠
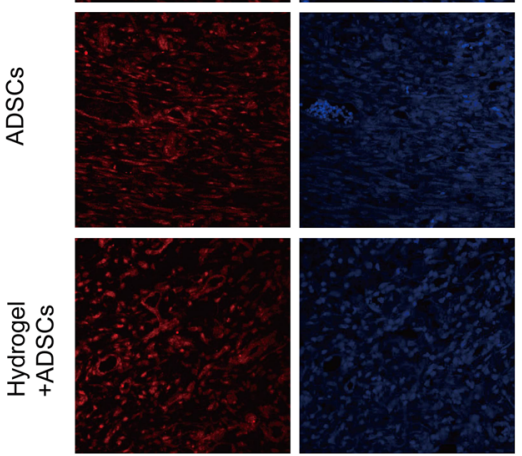

D

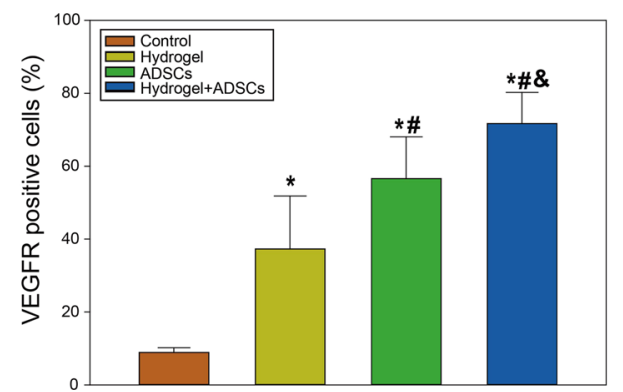

Merge
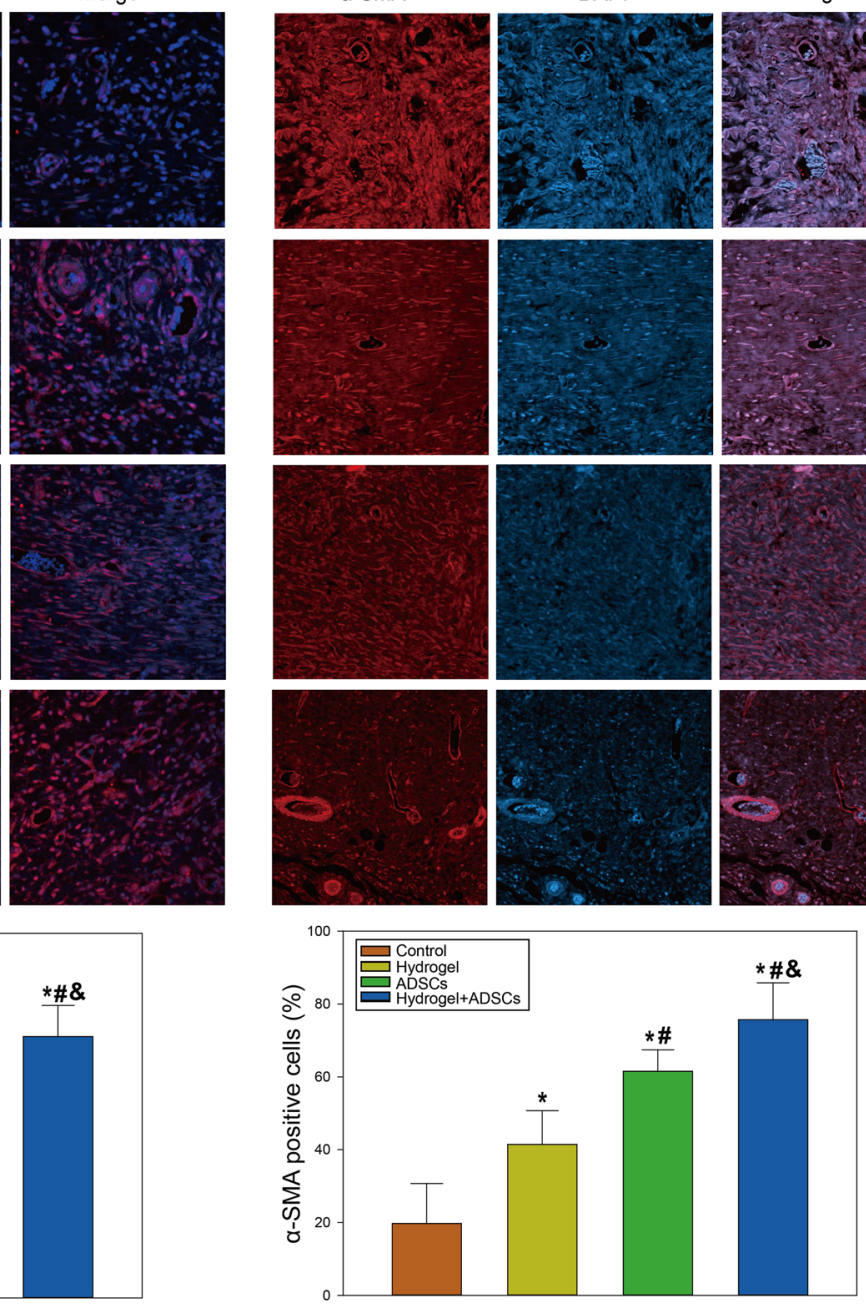
Fig. 5 ADSCs-loaded $\beta$-ChNF hydrogel accelerated angiogenesis and collagen deposition. A Representative western blot images of VEGFR, $\alpha$-SMA, collagen I, collagen III, and GAPDH. B Quantitative analysis of VEGFR, $\alpha$-SMA, collagen I and collagen III. Results are expressed as the mean \pm SD. ${ }^{*} P<0.05$, vs. the control group; ${ }^{\#} P<0.05$, vs. the ADSCs group; ${ }^{\&} P<0.05$, vs. the ADSCs + hydrogel group. C Representative immunofluorescent staining images showing the effect of ADSCs-loaded $\beta$-ChNF hydrogel on VEGFR and $\alpha$-SMA expression. D The quantitative analysis of VEGFR, $\alpha$-SMA. ${ }^{*} P<0.05$, vs. the control group; ${ }^{\#} P<0.05$, vs. the ADSCs group; ${ }^{\&} P<0.05$, vs. the ADSCs+hydrogel group

\section{Discussion}

In nature, chitin exists as different allomorphic forms that vary in terms of their polymer chain structure and crystallinity. $\alpha$-Chitin has a two-chain, antiparallel structure, and was the most abundant form of chitin found in insects and crustaceans, while the rarer $\beta$-chitin allomorph occurs in squid pens and some diatoms, and has a one-chain unit cell with a parallel-chain structure [24, 25]. Due to its good biocompatibility and high porosity similar to the skin's natural extracellular matrix, $\beta$-chitin nanofibers are conducive to cell adhesion and growth [26]. In this study, the squid cartilage was made into $\beta$-ChNF dispersion. We found that the $\beta$-ChNF dispersion was gelled upon the addition of cell culture medium, which was beneficial to its successful application. The weaker hydrogen-bonding of the parallel-chain structure of $\beta$-chitin may account for its higher chemical reactivity. In addition, $\beta$-chitin has the unique feature of incorporating small molecules, including water, into its crystal lattice to form crystalline complexes $[27,28]$. The unique structure of intramolecular hydrogen bonds might facilitate the combination of the active components of the culture medium to form a gel. The hydrogels exhibited a highly and interconnected porous structure, which was expected to have a high permeability for nutrients and to support cellular growth.

The cell culture experiments in this study further proved the properties of $\beta$-ChNFs hydrogel in cellular growth. In vitro tests have shown that $\beta$-ChNFs exhibited negligible cytotoxicity towards ADSCs and L929 cells, and the globular ADSCs were found in the $\beta$-ChNF hydrogel. The loose porous structure could be highly permeable to nutrients and promote the spread of cells seeded on the chitin nanofibrous matrices, as well as the formation of cell clusters that were tightly packed. Porous chitin nanofiber was therefore a suitable substrate for cell adhesion and supports stem cell self-renewal [29]. Furthermore, it was found that more exosomes were secreted by the ADSCs grown in the $\beta$-ChNF hydrogel. The secretion of exosomes is one of the ways that stem cells exert biological activity. Exosomes have a double-layer membrane rich in bioactive molecules, such as cholesterol, sphingolipids, ceramides, nucleic acids, proteins, carbohydrates and other molecules. They could target specific receptor cells and act as carriers for intercellular signal transduction [30]. In this study, ADSCs were located on the inner walls of the scaffold pores, proliferated and grew well in the three-dimensional porous structure. The viable cells were uniformly distributed in the $\beta$-ChNF hydrogel and more exosomes were efficiently secreted. These properties indicate that the support is a viable biomaterial for accelerating the wound healing, which can solve the problem of cell localization and survival.

Healing of open cutaneous wounds comprises a complex series of events, namely epithelialization, connective tissue deposition and contraction [31]. The existing methods for promoting wound healing have some problems such as limited effect or inconvenient use. Stem cell therapy has been shown to be effective in treating the chronic wound, and the scaffold for stem cell played an important role for its successful applications [32]. In this study, $\beta$-ChNF hydrogel was investigated as the scaffold of ADSCs. ADSCs-loaded $\beta$-ChNF hydrogel showed a positive effect in 2 days, and promoted rats wound healing in 10 days. $\beta$-ChNF hydrogel can provide a wet healing environment for wound healing. Good hydration is an important external factor that contributes to optimal wound healing, which can prevent undesirable scab formation as well as further dermal damage induced by desiccation [33]. The observed improvement in healing may be related to easier migration of epidermal cells over the moist wound surface rather than beneath a dry scab and the preservation of growth factors and proteinases present in fluid exudates that can then exert their potentiating effect on wound healing [34]. In addition to the above functions, the ADSCs-loaded $\beta$-ChNF hydrogel also allowed ADSCs to persist within the wound area and accelerated wound healing. ADSCs has been reported to enhance tissue regeneration via two different mechanisms, either by differentiating into skin cells or by secretion of paracrine factors [35]. It has been found that ADSCs can stimulate angiogenesis, epithelialization, and wound remodeling through paracrine secretion during wound repair. In rodent burn models, ADSCs modulated expression at the cellular level, specifically increased the expression of VEGF and collagens, which were related to microvessel formation and damage repair [36]. However, the ADSCs served to quell inflammation and stimulate angiogenesis was mostly applied by injections. The advantage of this experiment is that the ADSCs-loaded $\beta$-ChNF hydrogel could directly load on the wound surface and significantly accelerate wound healing by accelerating epitheliogenesis and promoting angiogenesis as well as collagen deposition. Furthermore, the better effects of ADSCs-loaded $\beta$-ChNF hydrogel during wound healing than ADSCs or hydrogel can be attributed to the healing 

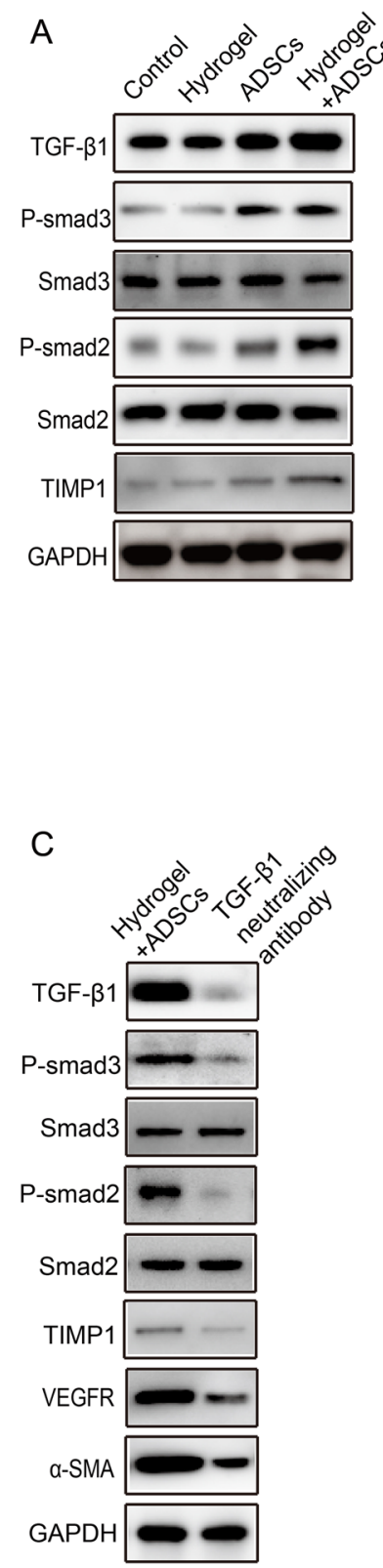
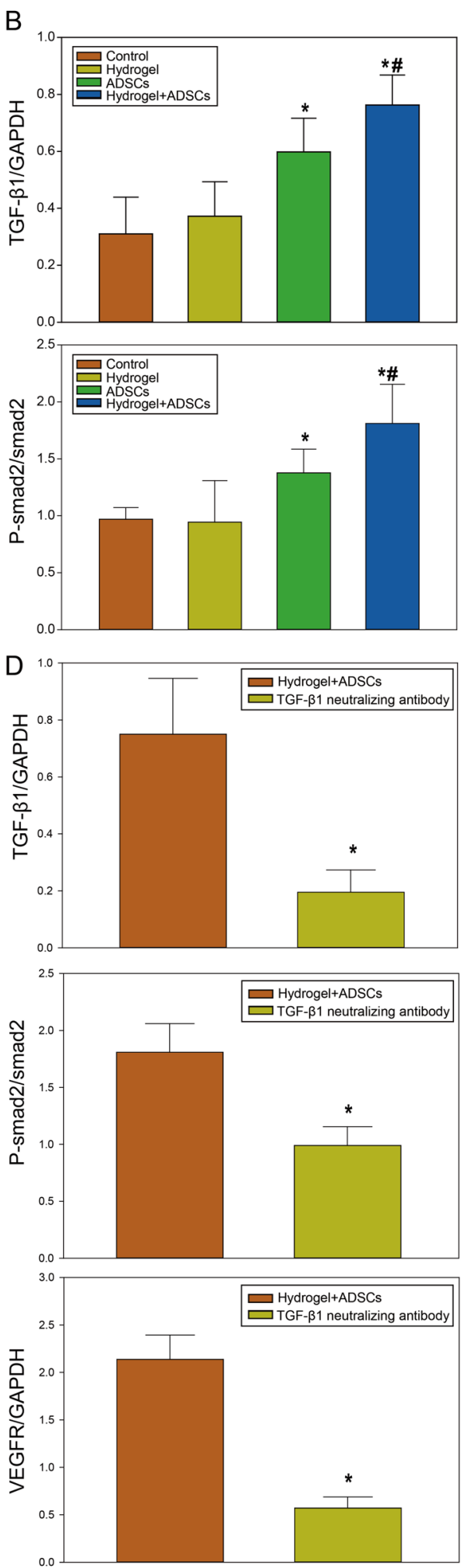
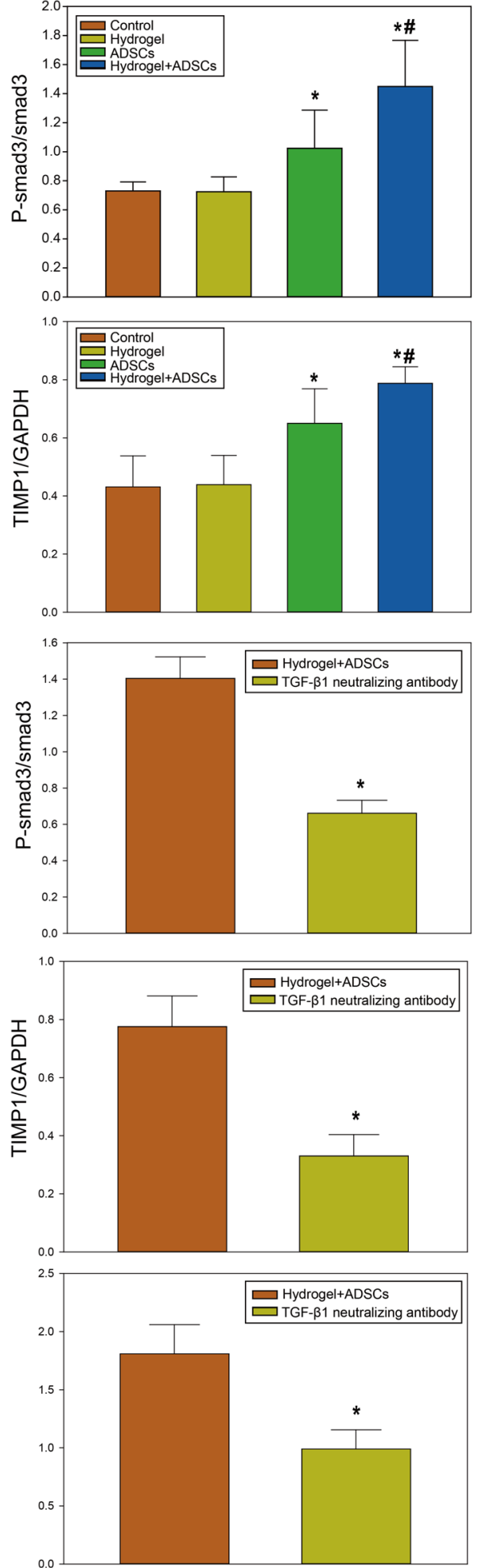

Fig. 6 ADSCs-loaded $\beta$-ChNF hydrogel regulated the TGF $\beta /$ smad3 signaling pathway. A Representative western blot images of TGF $\beta 1$, P-smad3, smad3, P-smad2, smad2, TIMP1, and GAPDH. B Quantitative analysis of TGF 1 , P-smad3, smad3, P-smad2, smad2, and TIMP1. Results are expressed as the mean \pm SD. ${ }^{*} P<0.05$, vs. the control group; ${ }^{\#} P<0.05$, vs. the ADSCs group; ${ }^{\circledR} P<0.05$, vs. the ADSCs ${ }^{+}$hydrogel group. C Representative western blot images of TGF 31 , P-smad3, smad3, P-smad2, smad2, TIMP1, and GAPDH after treatment with neutralizing antibody. D Quantitative analysis of TGF $\beta 1$, P-smad3, smad3, P-smad2, smad2, and TIMP1 after treatment with neutralizing antibody. Results are expressed as the mean \pm SD. ${ }^{*} P<0.05$, vs. the control group; ${ }^{*} P<0.05$, vs. the ADSCs group; ${ }^{\&} P<0.05$, vs. the ADSCs + hydrogel group 
properties of the ADSCs and also the three-dimensional environment of the hydrogel.

Collagens are the primary dermal matrix, which are responsible for the proliferation, tensile strength of skin and the formation of scars [13]. The ADSCs-loaded $\beta$-ChNF hydrogel could effectively secrete exosomes and accelerate angiogenesis and collagen deposition, while the specific mechanism required determination. TGF $\beta /$ Smad signaling pathway is related to the collagen formation during damage repair. Therefore, we observed the change of TGF $\beta$ / Smad signaling pathway in this study. It is reported that mesenchymal stem cell-derived exosomes microRNAs could adjust the TGF- $\beta$ /smad pathway during wound healing, which is consistent with our results [37]. TGF- $\beta /$ smad is involved in wound healing processes through neoangiogenesis [38]. TIMP1 plays a role in inhibiting MMP deposition and preventing ECM degradation. In addition, TIMP1 has been known to promote fibroblast proliferation and enhance new blood vessel formation [39-41]. This study showed that ADSCsloaded $\beta$-ChNF hydrogel treatment up-regulated the expression of TIMP1. Therefore, the success of $\beta$-chitin in this application is related to the provision of a threedimensional hydrogel microenvironment for ADSCs to promote angiogenesis through the TGF $\beta /$ smad signaling pathway.

\section{Conclusion}

In this study, squid pens were used to prepare $\beta$-chitin nanofiber dispersion, which gelled upon the addition of cell culture medium and created an appropriate microenvironment for cell adhesion and exosome secretion. The prepared hydrogel showed advantages as ADSC support and was demonstrated to be an effective carrier material for stem cells in the treatment of wounds. The mechanism of this study revealed that in addition to playing the role of hydrogel to accelerate epithelialization, ADSCs-loaded $\beta$-ChNF hydrogel could also significantly promote angiogenesis and collagen deposition through the TGF $\beta /$ smad3 signaling pathway. Our study demonstrates the potential of clinical applications of the stem cell-based $\beta$-chitin nanofiber hydrogels, which might be promising to be applied in the healing process of acute and chronic wounds.

\section{Data availability}

The datasets generated during and/or analyzed during the current study are available from the corresponding author on reasonable request.
Acknowledgements We thank Sarah Dodds, PhD, from Liwen Bianji, Edanz Editing China (www.liwenbianji.cn/ac), for editing the English text of a draft of this manuscript.

Author contributions HXJ, YLB, and YZ designed the experiments. MXH provided guidance. YL wrote the manuscript. YL, RFZ, SM, and PFC performed the experiments. MW completed the statistical analysis. All authors read and approved the final manuscript.

Funding This work was supported by the Department of Science and Technology of Liaoning Province under grant (No.2019010205), PLA Foundation of China under grant (No. AWS15J004-2-1).

\section{Compliance with ethical standards}

Conflict of interest The authors declare no competing interests.

Ethics statement Animal experiments were approved by the Ethics Committee of the General Hospital of the Northern Theater Command.

Publisher's note Springer Nature remains neutral with regard to jurisdictional claims in published maps and institutional affiliations.

Open Access This article is licensed under a Creative Commons Attribution 4.0 International License, which permits use, sharing, adaptation, distribution and reproduction in any medium or format, as long as you give appropriate credit to the original author(s) and the source, provide a link to the Creative Commons license, and indicate if changes were made. The images or other third party material in this article are included in the article's Creative Commons license, unless indicated otherwise in a credit line to the material. If material is not included in the article's Creative Commons license and your intended use is not permitted by statutory regulation or exceeds the permitted use, you will need to obtain permission directly from the copyright holder. To view a copy of this license, visit http://creativecommons. org/licenses/by/4.0/.

\section{References}

1. Shen T, Dai K, Yu Y, Wang J, Liu C. Sulfated chitosan rescues dysfunctional macrophages and accelerates wound healing in diabetic mice. Acta Biomater. 2020;117:192-203. https://doi.org/ 10.1016/j.actbio.2020.09.035

2. Lee YH, Hong YL, Wu TL. Novel silver and nanoparticleencapsulated growth factor co-loaded chitosan composite hydrogel with sustained antimicrobility and promoted biological properties for diabetic wound healing. Mater Sci Eng C Mater Biol Appl. 2021;118:111385. https://doi.org/10.1016/j.msec.2020. 111385

3. Hanafy MS, Desoky WM, Hussein EM, El-Shaer NH, Gomaa M, Gamal AA, et al. Biological applications study of bionanocomposites based on chitosan/TiO2 nanoparticles polymeric films modified by oleic acid. J Biomed Mater Res A. 2021;109:232-47. https://doi.org/10.1002/jbm.a.37019

4. Hosseini Mansoub N, Gürdal M, Karadadaş E, Kabadayi H, Vatansever S, Ercan G. The role of PRP and adipose tissuederived keratinocytes on burn wound healing in diabetic rats. Bioimpacts. 2018;8:5-12. https://doi.org/10.15171/bi.2018.02

5. Hassan WU, Greiser U, Wang W. Role of adipose-derived stem cells in wound healing. Wound Repair Regen. 2014;22:313-25. https://doi.org/10.1111/wrr.12173 
6. Jafari H, Bernaerts KV, Dodi G, Shavandi A. Chitooligosaccharides for wound healing biomaterials engineering. Mater Sci Eng C Mater Biol Appl. 2020;117:111266. https://doi. org/10.1016/j.msec.2020.111266

7. Laloze J, Fiévet L, Desmoulière A. Adipose-derived mesenchymal stromal cells in regenerative medicine: state of play, current clinical trials, and future prospects. Adv Wound Care (N. Rochelle). 2021;10:24-48. https://doi.org/10.1089/wound.2020.1175

8. Raghunathan VK, Thomasy SM, Strøm P, Yañez-Soto B, Garland SP, Sermeno J, et al. Tissue and cellular biomechanics during corneal wound injury and repair. Acta Biomater. 2017;58:291-301. https://doi.org/10.1016/j.actbio.2017.05.051

9. Li X, Guo W, Zha K, Jing X, Wang M, Zhang Y, et al. Enrichment of CD146+adipose-derived stem cells in combination with articular cartilage extracellular matrix scaffold promotes cartilage regeneration. Theranostics 2019;9:5105-21. https://doi.org/10. 7150/thno. 33904

10. Gleeson BM, Martin K, Ali MT, Kumar AH, Pillai MG, Kumar $\mathrm{SP}$, et al. Bone marrow-derived mesenchymal stem cells have innate procoagulant activity and cause microvascular obstruction following intracoronary delivery: amelioration by antithrombin therapy. Stem Cells. 2015;33:2726-37. https://doi.org/10.1002/ stem.2050

11. Mazini L, Rochette L, Admou B, Amal S, Malka G. Hopes and limits of adipose-derived stem cells (ADSCs) and mesenchymal stem cells (MSCs) in wound healing. Int J Mol Sci. 2020;21:1306. https://doi.org/10.3390/ijms21041306

12. Klar AS, Zimoch J, Biedermann T. Skin tissue engineering: application of adipose-derived stem cells. Biomed Res Int. 2017;2017:9747010. https://doi.org/10.1155/2017/9747010

13. Wei X, Liu B, Liu G, Yang F, Cao F, Dou X, et al. Mesenchymal stem cell-loaded porous tantalum integrated with biomimetic 3D collagen-based scaffold to repair large osteochondral defects in goats. Stem Cell Res Ther. 2019;10:72. https://doi.org/10.1186/ s13287-019-1176-2

14. Ahsan SM, Thomas M, Reddy KK, Sooraparaju SG, Asthana A, Bhatnagar I. Chitosan as biomaterial in drug delivery and tissue engineering. Int J Biol Macromol. 2018;110:97-109. https://doi. org/10.1016/j.ijbiomac.2017.08.140

15. Lei Z, Singh G, Min Z, Shixuan C, Xu K, Pengcheng X, et al. Bone marrow-derived mesenchymal stem cells laden novel thermo-sensitive hydrogel for the management of severe skin wound healing. Mater Sci Eng C Mater Biol Appl. 2018;90:159-67. https://doi.org/10.1016/j.msec.2018.04.045

16. Wan AC, Tai BC. CHITIN-a promising biomaterial for tissue engineering and stem cell technologies. Biotechnol Adv. 2013;31:1776-85. https://doi.org/10.1016/j.biotechadv.2013.09.007

17. Khil MS, Cha DI, Kim HY, Kim IS, Bhattarai N. Electrospun nanofibrous polyurethane membrane as wound dressing. J Biomed Mater Res B Appl Biomater. 2003;67:675-9. https://doi.org/10. 1002/jbm.b.10058

18. Shou K, Huang Y, Qi B, Hu X, Ma Z, Lu A, et al. Induction of mesenchymal stem cell differentiation in the absence of soluble inducer for cutaneous wound regeneration by a chitin nanofiberbased hydrogel. J Tissue Eng Regen Med. 2018;12:e867-e880. https://doi.org/10.1002/term.2400

19. Liang Y, Zhao X, Hu T, Chen B, Yin Z, Ma PX, et al. Adhesive hemostatic conducting injectable composite hydrogels with sustained drug release and photothermal antibacterial activity to promote full-thickness skin regeneration during wound healing. Small. 2019;15:e1900046. https://doi.org/10.1002/smll.201900046

20. Lavall RL, Assis OB, Campana-Filho SP. Beta-chitin from the pens of Loligo sp.: extraction and characterization. Bioresour Technol. 2007;98:2465-72. https://doi.org/10.1016/j.biortech. 2006.09.002
21. Nata IF, Wang SS, Wu TM, Lee CK. $\beta$-Chitin nanofibrils for selfsustaining hydrogels preparation via hydrothermal treatment. Carbohydr Polym. 2012;90:1509-14. https://doi.org/10.1016/j.ca rbpol.2012.07.022

22. Subin S, Yan Z, Xiaoxue Y, Jinsong Z. $\beta$-chitin nanofiber hydrogel as a scaffold to in situ fabricate monodispersed ultrasmall silver nanoparticles. Colloid Surf A. 2019;574:36-43. https://doi.org/10.1016/j.colsurfa.2019.04.047

23. Noishiki Y, Takami H, Nishiyama Y, Wada M, Okada S, Kuga S. Alkali-induced conversion of beta-chitin to alpha-chitin. Biomacromolecules 2003;4:896-9. https://doi.org/10.1021/bm0257513

24. Balitaan JNI, Hsiao CD, Yeh JM, Santiago KS. Innovation inspired by nature: Biocompatible self-healing injectable hydrogels based on modified- $\beta$-chitin for wound healing. Int $\mathrm{J}$ Biol Macromol. 2020;162:723-36. https://doi.org/10.1016/j.ijbiomac. 2020.06.129

25. Jayakumar R, Divya Rani VV, Shalumon KT, Kumar PT, Nair $\mathrm{SV}$, Furuike T, et al. Bioactive and osteoblast cell attachment studies of novel alpha- and beta-chitin membranes for tissueengineering applications. Int J Biol Macromol. 2009;45:260-4. https://doi.org/10.1016/j.ijbiomac.2009.06.002

26. Jones M, Kujundzic M, John S, Bismarck A. Crab vs. mushroom: a review of crustacean and fungal chitin in wound treatment. Mar Drugs. 2020;18:64. https://doi.org/10.3390/md18010064

27. Ahmad SI, Ahmad R, Khan MS, Kant R, Shahid S, Gautam L, et al. Chitin and its derivatives: structural properties and biomedical applications. Int J Biol Macromol. 2020;164:526-39. https:// doi.org/10.1016/j.ijbiomac.2020.07.098

28. Ifuku S. Chitin and chitosan nanofibers: preparation and chemical modifications. Molecules. 2014;19:18367-80. https://doi.org/10. 3390/molecules 191118367

29. Song K, Ji L, Zhang J, Wang H, Jiao Z, Mayasari L, et al. Fabrication and cell responsive behavior of macroporous PLLA/ gelatin composite scaffold with hierarchical micro-nano pore structure. Nanomaterials (Basel) 2015;5:415-24. https://doi.org/ 10.3390/nano5020415

30. Zhang Y, Han F, Gu L, Ji P, Yang X, Liu M, et al. Adipose mesenchymal stem cell exosomes promote wound healing through accelerated keratinocyte migration and proliferation by activating the AKT/HIF- $1 \alpha$ axis. J Mol Histol. 2020;51:375-83. https://doi. org/10.1007/s10735-020-09887-4

31. Zhao X, Wu H, Guo B, Dong R, Qiu Y, Ma PX. Antibacterial anti-oxidant electroactive injectable hydrogel as self-healing wound dressing with hemostasis and adhesiveness for cutaneous wound healing. Biomaterials. 2017;122:34-47. https://doi.org/10. 1016/j.biomaterials.2017.01.011

32. Abolgheit S, Abdelkader S, Aboushelib M, Omar E, Mehanna R. Bone marrow-derived mesenchymal stem cells and extracellular vesicles enriched collagen chitosan scaffold in skin wound healing (a rat model). J Biomater Appl. 2021;36:128-39. https://doi.org/ $10.1177 / 0885328220963920$

33. Zhao CC, Zhu L, Wu Z, Yang R, Xu N, Liang L. Resveratrolloaded peptide-hydrogels inhibit scar formation in wound healing through suppressing inflammation. Regen Biomater. 2020;7:99-107. https://doi.org/10.1093/rb/rbz041

34. Tchemtchoua VT, Atanasova G, Aqil A, Filée P, Garbacki N, Vanhooteghem O, et al. Development of a chitosan nanofibrillar scaffold for skin repair and regeneration. Biomacromolecules. 2011;12:3194-204. https://doi.org/10.1021/bm200680q

35. Li Q, Xia S, Yin Y, Guo Y, Chen F, Jin P. miR-5591-5p regulates the effect of ADSCs in repairing diabetic wound via targeting AGEs/AGER/JNK signaling axis. Cell Death Dis. 2018;9:566. https://doi.org/10.1038/s41419-018-0615-9

36. Liu L, Yu Y, Hou Y, Chai J, Duan H, Chu W, et al. Human umbilical cord mesenchymal stem cells transplantation promotes 
cutaneous wound healing of severe burned rats. PLoS One. 2014;9:e88348. https://doi.org/10.1371/journal.pone.0088348

37. Jiang T, Wang Z, Sun J. Human bone marrow mesenchymal stem cell-derived exosomes stimulate cutaneous wound healing mediates through TGF- $\beta /$ Smad signaling pathway. Stem Cell Res Ther. 2020;11:198. https://doi.org/10.1186/s13287-02001723-6

38. Hwang E, Lin P, Ngo HTT, Yi TH. Clove attenuates UVBinduced photodamage and repairs skin barrier function in hairless mice. Food Funct. 2018;9:4936-47. https://doi.org/10.1039/ c8fo00843d

39. Liu H, Chen B, Lilly B. Fibroblasts potentiate blood vessel formation partially through secreted factor TIMP-1.
Angiogenesis. 2008;11:223-34. https://doi.org/10.1007/ s10456-008-9102-8

40. Wang L, Hu L, Zhou X, Xiong Z, Zhang C, Shehada HMA, et al. Exosomes secreted by human adipose mesenchymal stem cells promote scarless cutaneous repair by regulating extracellular matrix remodelling. Sci Rep. 2017;7:13321. https://doi.org/10. 1038/s41598-017-12919-x

41. Kananykhina EY, Shmakova TV, Bolshakova GB, Rusanov FS, Elchaninov AV, Nikitina MP, et al. Expression of metalloproteinases and type I and III collagens during healing of excisional skin wound on the abdomen and back in rats. Bull Exp Biol Med. 2020;168:812-6. https://doi.org/10.1007/s10517-02004808-7 